\title{
Hyperiid Amphipods (Crustacea: Peracarida) in Mexican Waters of the Pacific Ocean ${ }^{1}$
}

\author{
Rebeca Gasca ${ }^{2}$
}

\begin{abstract}
Information on regional diversity of hyperiid amphipods of the eastern Pacific Ocean is still largely incomplete. Recent surveys of hyperiid fauna from the Mexican Pacific motivated a revision of extant faunistic accounts. This revised list includes all records from Mexican waters of the eastern Pacific (MP) from Baja California to the southern border with Central America. A total of 150 species belonging to 19 families and 48 genera of the Hyperiidea was included in this account; seven are new records in the MP. Up to 31 nominal species were excluded from previous listings. Overall, the epipelagic infraorder Physocephalata is highly diverse in the MP (119 species); Physosomata, containing deep-living forms, are less diverse (31 species). The northern part of the MP (including the gulf and off the Baja California peninsula) harbors the highest number of species/records, whereas nearly half of the species are known from the central areas and six from the southernmost sector of the MP. This pattern reflects current knowledge of the group in these areas and also geographic differences in the sampling/research efforts, but it is not a diversity gradient. Species richness of the MP is comparable with that known from other Pacific subregions. The epipelagic hyperiid fauna of the tropical MP remains relatively unknown and should be studied further to reveal regional patterns of diversity. The deep-living hyperiid community of the tropical eastern Pacific harbors a diversity that is deserving of further study.
\end{abstract}

Hyperidd Amphipods represent one of the most abundant and diverse groups of crustaceans in the pelagic realm; this taxon contains more than 250 species, many of them with a wide geographic distribution (Vinogradov et al. 1996, Vinogradov 1999). Most of these forms are known to be symbiotically associated with different groups of gelatinous zooplankton (Laval 1980).

1 The University of Guadalajara supported part of this research and the sampling logistics to survey the coasts of Jalisco and Colima through the project "Demersales." This contribution is part of the requirements of the postgraduate program of the University of Guadalajara. Manuscript accepted 4 April 2008.

${ }^{2}$ El Colegio de la Frontera Sur (ECOSUR), Unidad Chetumal, Apartado Postal 424, Chetumal, Quintana Roo 77000, Mexico (e-mail: rgasca@ecosur.mx).

Pacific Science (2009), vol. 63, no. 1:83-95

(C) 2009 by University of Hawai'i Press

All rights reserved
There are large oceanic and neritic areas in which the diversity of this group remains practically unknown, and the local faunal composition is only extrapolated from that known in adjacent areas (Gasca 2007). In other regions, such as the eastern Pacific, most of the available information is based on isolated works developed over the decades, some of them including Mexican Pacific waters. Despite the amount of data from these sources, some of these checklists have not been critical, and unrevised records are repeated from previous works, particularly in Mexican waters. Many of these records are outdated or invalid. There have been no recent efforts to produce an updated, critical checklist of the hyperiid amphipods from oceanic and coastal areas of the Mexican Pacific.

The regional works in which reference is made to the hyperiid amphipods of Mexican waters of the Pacific Ocean (MP) fall into two categories: (1) those in which original records/observations are presented by the authors, and (2) those in which records are tak- 
en from previous works or accounts, without comments or additions. Among the works in the first category, the oldest ones are those by Streets (1878), who recorded Rhabdosoma whitei Bate, 1862, and Shoemaker (1925), who recorded nine species collected during a cruise of the Albatross in the Gulf of California; he described three new species. Later, Hurley (1956) provided records of 34 hyperiids from Californian waters including 19 species found in Mexican waters $\left(17^{\circ} 50^{\prime} \mathrm{N}\right)$, and Bowman (1960) recorded Themisto pacifica (Stebbing, 1888) off Baja California. Bowman (1973) studied the different genera of the Hyperiidae from the eastern Pacific, covering also waters off Baja California. Brusca (1981) published a commented key to the hyperiids of the North American coastal waters that included distributional observations of many species and records from the MP. The monographic account by Siegel-Causey (1982) was focused on the hyperiid fauna of the Gulf of California, where he recorded 118 species. Lavaniegos and Ohman (1999) studied the hyperiids of the California Current subregion and did not include data from Mexican waters; however, Ohman and Lavaniegos (2002) included records from southern California and Baja California. Shih and Hendrycks (2003) studied the genus Vibilia from an extensive area of the eastern tropical $\mathrm{Pa}$ cific, including Mexican waters. Gasca and Haddock (2004) provided new records of symbiotic associations of hyperiid species from the gulf, and Gasca (2005) described a new deep-living species of Hyperoche. More recently, Gasca and Franco-Gordo (2008) surveyed the hyperiid fauna from Banderas Bay, a coastal system in the Mexican tropical Pacific.

Among the contributions of the second category, the work by Escobar-Briones et al. (2002) is included. The list comprised all the amphipods (benthic and pelagic) recorded in Mexican waters. However, records were not assigned to a region; these were summarized into a single generalized account. Hence, the information about the records from the MP is unavailable. Escobar-Briones and Winfield (2003) provided a list of the amphipods from the Mexican Pacific, including an account of the hyperiids. Later, Brusca and Hendrickx (2005) published a list of the peracarid crustaceans with a section about the hyperiids from the Gulf of California. Brinton et al. (1986) analyzed the distribution of some hyperiids in the gulf based on data by Siegel-Causey (1982). The species recorded in the work by Brusca (1967) off California were probably included in the general accounts from Mexican waters; however, the survey is limited to non-Mexican waters of the Californian region. Based on records from the literature and on original data, a new list is presented herein. It is also recognized that local and regional surveys of the diversity and/or biogeography of the group must be anchored on reliable taxonomic lists.

\section{MATERIALS AND METHODS}

The list of species presented here was obtained from analysis of the extant literature about the hyperiid amphipods from Mexican waters of the Pacific Ocean; records from adjacent areas were not included except for data from sampling sites located within the MP or from immediate adjacent zones. Original and new observations/data from tropical waters off the coasts of Jalisco and Nayarit (Gasca and Franco-Gordo 2008) and personal observations based on samples collected off the coasts of Jalisco and Colima (1995-1997) were also included in this account. Records were sorted considering three sectors of the MP: the Gulf of California, Baja California, and the central and southern areas of the MP (Figure 1).

The taxonomic arrangement used in this account followed different sources, depending on the family: Vinogradov et al. (1996) was used as the basic work for the identification or classification of several genera. The nomenclature and criteria proposed by Harbison and Madin (1976) were followed in reference to species of Lycaea. The work by Shih (1991) was used to identify and arrange the species of Phronima. For the superfamilies Vibilioidea, Lycaepsoidea, Phronimoidea, and Archaeoscinoidea, the recent works by Zei- 


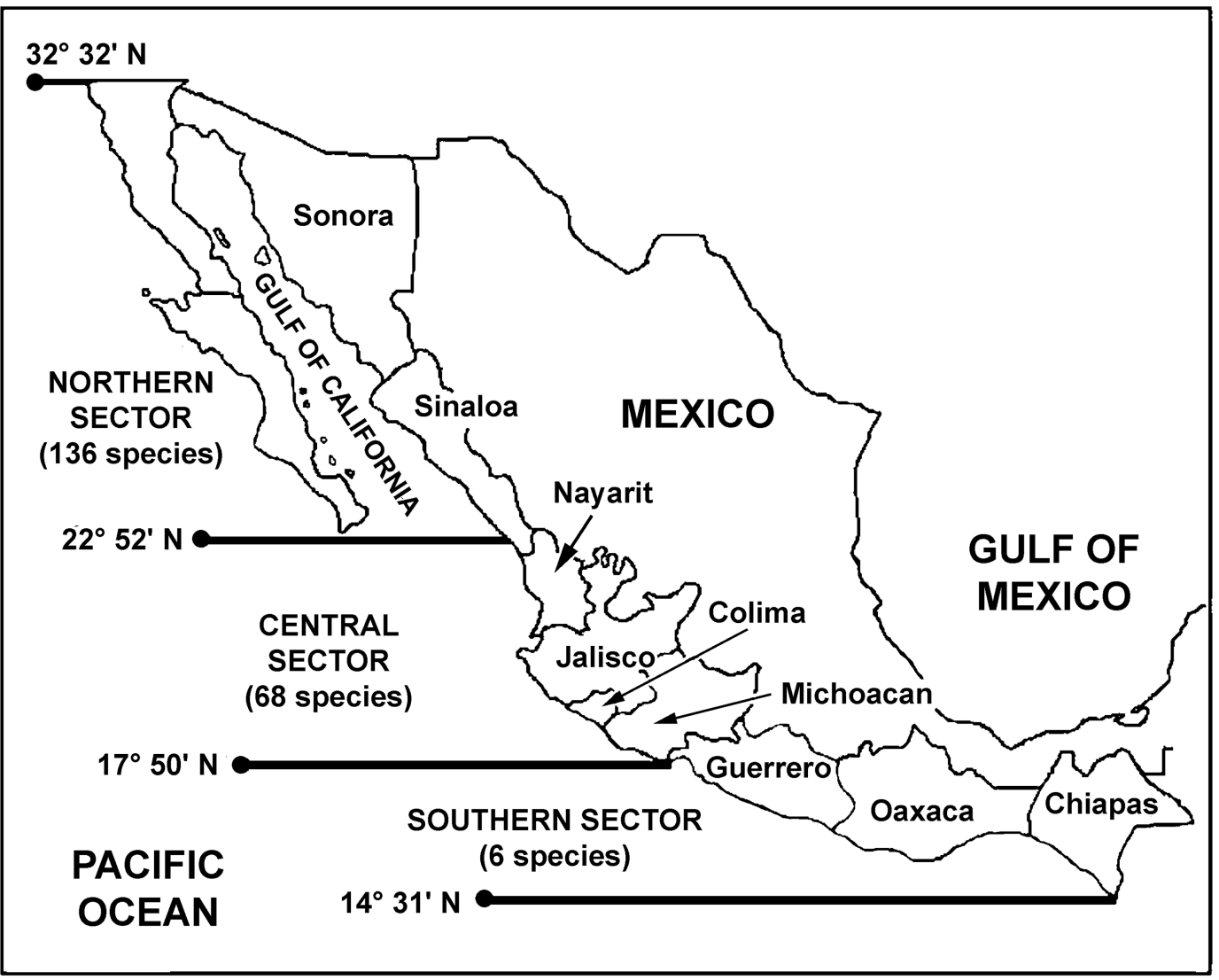

Figure 1. Latitudinal sectors of the Mexican waters of the Pacific Ocean showing the states of the Mexican Pacific coast and the number of species of Hyperiidae recorded from each sector.

dler (2003a, 2004a,b, 2006) were used. Also, Zeidler (1999) was followed for species of Oxycephalus, and Zeidler (2003b) in reference to the Cystisomatidae.

The synonymies of each species contained in this checklist were revised here, most based on Shih and Cheng (1995) and Vinogradov et al. (1996). Some species mentioned in other lists under a different name were included in this account with the new, accepted name. Some of the species collected in this survey have not been hitherto recorded in the Mexican Pacific; voucher specimens of these species were deposited in the collection of zooplankton held at El Colegio de la Frontera Sur, Chetumal, Mexico (ECO-CHz).
RESULTS

The checklist presented herein contains a total of 150 species of hyperiid amphipods confirmed from Mexican waters. This figure includes representatives of the two infraorders of the Hyperiidea, the Physosomata and the Physocephalata. The former group is represented by 31 species, the latter by 119 . Overall, the list includes 19 families and 48 genera. At the genus level the most diverse families were Oxycephalidae (7), Lestrigonidae (5), and Platyscelidae (5). The most speciose family in the MP was the Scinidae (22), followed by Lestrigonidae (15), Vibiliidae (14), and Platyscelidae (13) (Table 1). 
TABLE 1

Updated Checklist of the Hyperiid Amphipods from the Mexican Waters of the Pacific Ocean

\begin{tabular}{|c|c|c|}
\hline Taxa & $\begin{array}{l}\text { Records from the } \\
\text { Gulf of California }{ }^{a}\end{array}$ & $\begin{array}{l}\text { Records from } \\
\text { the Pacific Coast }\end{array}$ \\
\hline \multicolumn{3}{|l|}{ Order Amphipoda } \\
\hline \multicolumn{3}{|l|}{ Suborder Hyperiidea } \\
\hline \multicolumn{3}{|l|}{ Infraorder Physosomata Pirlot, 1929} \\
\hline \multicolumn{3}{|l|}{ Superfamily Lanceoloidea Bovallius, 1887} \\
\hline \multicolumn{3}{|l|}{ Family Lanceolidae Bovallius, 1887} \\
\hline Lanceola loveni Bovallius, 1885 & $\mathrm{SC} 82^{1}, \mathrm{BH} 05$ & EBW03 \\
\hline Lanceola sayana Bovallius, 1885 & SC82, BH05 & EBW03 \\
\hline Lanceola pacifica Stebbing, 1888 & $\mathrm{SC} 82, \mathrm{BH} 05$ & EBW03 \\
\hline Lanceola loveni loveni Bovallius, 1882 & & $\mathrm{Hu} 56^{2}$ \\
\hline Lanceola loveni grossipes Shoemaker, 1945 & & EBW03 \\
\hline Scypholanceola aestiva (Stebbing, 1988) & SC82, BH05 & OL02, EBW03 \\
\hline Scypholanceola agassizi Woltereck, 1909 & $\mathrm{SC} 82, \mathrm{BH} 05$ & EBW03 \\
\hline \multicolumn{3}{|l|}{ Superfamily Scinoidea Stebbing, 1888} \\
\hline \multicolumn{3}{|l|}{ Family Mimonectidae Bovallius, 1885} \\
\hline Mimonectes gaussi (Woltereck, 1904) & SC82 & EBW03 \\
\hline Mimonectes diomedeae (Woltereck, 1909) & BH05 & EBW03 \\
\hline \multicolumn{3}{|l|}{ Family Scinidae Prestandrea, 1833} \\
\hline Scina crassicornis (Fabricius, 1775) & SC82, BH05 & EBW03 \\
\hline Scina curvidactyla Chevreux, 1914 & & EBW03 \\
\hline Scina incerta Chevreux, 1900 & & EBW03 \\
\hline Scina langhansi Wagler, 1926 & SC82, BH05, & EBW03 \\
\hline Scina borealis (G. O. Sars, 1882) & SC82, Bri86, BH05 & $\begin{array}{l}\text { Hu56, OL02, } \\
\text { EBW03 }\end{array}$ \\
\hline Scina spinosa Vosseler, 1901 & SC82, BH05 & EBW03 \\
\hline Scina stebbingi Chevreux, 1919 & SC82, BH05 & EBW03 \\
\hline Scina marginata (Bovallius, 1885) & SC82, BH05 & EBW03, PO \\
\hline Scina submarginata Tattersall, 1906 & SC82, BH05 & EBW03 \\
\hline Scina rattrayi rattrayi Stebbing, 1895 & $\mathrm{BH} 05$ & EBW03 \\
\hline Scina rattrayi keilhacki Uaeler, 1926 & SC82 & EBW03 \\
\hline Scina wolterecki Wagler, 1926 & SC82, BH05 & EBW03 \\
\hline Scina tullbergi (Bovallius, 1885) & & $\begin{array}{l}\text { Hu56, OL02, } \\
\text { EBW03 }\end{array}$ \\
\hline Scina similis Stebbing, 1895 & SC82, BH05 & EBW03 \\
\hline Scina nana Wagler, 1926 & SC82, BH05 & EBW03 \\
\hline Scina setigera Wagler, 1926 & SC82?, BH05 & EBW03 \\
\hline Scina excisa Wagler, 1926 & SC82, BH05 & EBW03 \\
\hline Scina damasi Pirlot, 1929 & $\mathrm{SC} 82, \mathrm{BH} 05$ & EBW03 \\
\hline Scina latifrons Wagler, 1926 & SC82, BH05 & EBW03 \\
\hline Scina pusilla Chevreux, 1919 & SC82, BH05 & EBW03 \\
\hline Scina inermis Chevreux, 1919 & & EBW03 \\
\hline Acanthoscina acanthodes (Stebbing, 1895) & $\mathrm{SC} 82, \mathrm{BH} 05$ & EBW03 \\
\hline \multicolumn{3}{|l|}{ Infraorder Physocephalata Bowman \& Gruner, 1973} \\
\hline \multicolumn{3}{|l|}{ Superfamily Vibilioidea Bowman \& Gruner, 1973} \\
\hline \multicolumn{3}{|l|}{ Family Vibiliidae Dana, 1852} \\
\hline Vibilia borealis Bate \& Westwood, 1868 & & EBW03, PO \\
\hline Vibilia gibbosa Bovallius, 1887 & & $\begin{array}{l}\mathrm{Hu} 56, \mathrm{OL} 02, \\
\text { EBW03 }\end{array}$ \\
\hline Vibilia robusta Bovallius, 1887 & & Bru81, EBW03 \\
\hline Vibilia viatrix Bovallius, 1887 & $\mathrm{Sho} 25^{3}, \mathrm{SC} 82, \mathrm{BH} 05$ & $\mathrm{Hu} 56, \mathrm{EBW} 03$ \\
\hline Vibilia armata Bovallius, 1887 & SC82, Bri86, BH05 & $\begin{array}{l}\mathrm{Hu} 56, \mathrm{OL} 02, \\
\text { EBW03, SH03, } \\
\text { PO }\end{array}$ \\
\hline Vibilia pyripes Bovallius, 1887 & & $\begin{array}{l}\text { Bru81, SH03, } \\
\text { EBW03 }\end{array}$ \\
\hline
\end{tabular}


TABLE 1 (continued)

\begin{tabular}{|c|c|c|c|}
\hline Taxa & & $\begin{array}{l}\text { Records from the } \\
\text { Gulf of California }{ }^{a}\end{array}$ & $\begin{array}{l}\text { Records from } \\
\text { the Pacific Coast }{ }^{a}\end{array}$ \\
\hline & Vibilia propinqua Stebbing, 1888 & SC82, SH03, BH05 & $\begin{array}{l}\text { Bru81, OL02, } \\
\text { EBW03, SH03, } \\
\text { PO }\end{array}$ \\
\hline & Vibilia australis Stebbing, 1888 & $\mathrm{SC}_{2}{ }^{4}, \mathrm{GH} 04^{4}$ & $\begin{array}{l}\text { Bru81 } 1^{5}, \mathrm{OL} 02, \\
\quad \mathrm{EBW} 03^{5}, \mathrm{SH} 03\end{array}$ \\
\hline & Vibilia antarctica Stebbing, 1888 & $\mathrm{SC} 82, \mathrm{BH} 05$ & EBW03 \\
\hline & Vibilia cultripes Vosseler, 1901 & SC82, BH05 & $\begin{array}{l}\text { Bru81, EBW03, } \\
\text { SH03 }\end{array}$ \\
\hline & Vibilia stebbingi Behning \& Woltereck, 1912 & SC82, BH05 & $\begin{array}{l}\text { Bru81, OL02, SH03, } \\
\text { EBW03 }\end{array}$ \\
\hline & Vibilia chuni Behning \& Woltereck, 1912 & SC82, BH05 & $\begin{array}{l}\text { Bru81, OL02, } \\
\text { EBW03, SH03, } \\
\text { PO }\end{array}$ \\
\hline
\end{tabular}

Vibilia wolterecki Behning, 1939

Vibilia longicarpus Behning, 1913

Family Paraphronimidae Bovallius, 1887

Paraphronima gracilis Claus, 1879

$\mathrm{SH} 03$

Paraphronima crassipes Claus, 1879

Superfamily Cystisomatoidea Zeidler, 2003

Family Cystisomatidae Willemöes-Suhm, 1875

Cystisoma fabricii Stebbing, 1888

Superfamily Phronimoidea Bowman \& Gruner, 1973

Family Phronimidae Dana, 1852

Phronima sedentaria (Forsskål, 1775)

Phronima atlantica Guérin-Méneville, 1836

Pbronima solitaria Guérin-Méneville, 1836

Phronima stebbingi Vosseler, 1901

Phronima curvipes Vosseler, 1901

Phronima colletti Bovallius, 1887

Phronima pacifica Streets, 1877

Phronima bucephala Giles, 1887

SC82, BH05

SC82, BH05

$\mathrm{SH} 03, \mathrm{PO}$

Bru81, OL02, EBW03, PO

OL02, EBW03

SC82, BH05

Hu56, EBW03

Phronima bowmani Shih, 1991

Pbronima dunbari Shih, 1991

Pbronimella elongata (Claus, 1862)

Family Phrosinidae Dana, 1852

Phrosina semilunata Risso, 1822

SC82, BH05

SC82, Bri86, BH05

SC82, BH05

SC82, Bri86, BH05

SC82, BH05

$\mathrm{SC}^{6} 2^{6}, \mathrm{Bri}^{6} 6^{6}$ BH05

$\mathrm{SC}^{7} 2^{7}, \mathrm{Bri}^{7} 6^{7}$,

BH05

SC82, Bri86, BH05

SC82, Bri86, BH05

Anchylomera blossevillei Milne-Edwards, 1830

Sho25, SC82, BH05

Primno macropa Guérin-Méneville, 1836

Primno brevidens Bowman, 1978

SC82, Bri86, BH05

Primno latreillei Stebbing, 1888

Family Hyperiidae Dana, 1852

Hyperia medusarum (Müller, 1776)

Hyperia galba (Montagu, 1815)

Hyperia spinigera Bovallius, 1889

Hyperia leptura Bowman, 1973

SC82, BH05

Hu56, Bru81, OL02, EBW03

Bru81, EBW03, PO

EBW03

OL02, EBW03

EBW03

Hu56, EBW03

OL02, EBW03

OL02, EBW03, GFG08, PO

Bru816 ${ }^{6}$ EBW03, PO

Bru817 ${ }^{7}$ EBW03, PO

EBW03, PO

Hu56, OL02,

EBW03, PO

Hu56, Bru81, EBW03, PO

EBW03

Bow78, Bru81, OL02, EBW03

Bow78, Bru81, OL02, EBW03, GFG08

Bow $73^{8}$, OL02, EBW03

EBW039

Bow73, Bru81

Bow73, EBW03 
TABLE 1 (continued)

\begin{tabular}{|c|c|c|}
\hline Taxa & $\begin{array}{l}\text { Records from the } \\
\text { Gulf of California }^{a}\end{array}$ & $\begin{array}{l}\text { Records from } \\
\text { the Pacific Coast }{ }^{a}\end{array}$ \\
\hline Themisto pacifica (Stebbing, 1888) & & $\begin{array}{l}\text { Bru81 }{ }^{10}, \text { OL02, } \\
\text { EBW03, Bow60 }\end{array}$ \\
\hline Hyperoche medusarum (Kröyer, 1838) & SC82, BH05 & $\begin{array}{l}\text { Bru81, OL02, } \\
\text { EBW03 }\end{array}$ \\
\hline Hyperoche martinezii (Müller, 1864) & SC82, BH05 & OL02, EBW03 \\
\hline Hyperoche picta Bovallius, 1889 & & GFG08 \\
\hline Hyperoche mediterranea Senna, 1908 & & $\begin{array}{l}\text { Bru81, OL02, } \\
\text { EBW03 }\end{array}$ \\
\hline Hyperoche shibi Gasca, 2005 & GH0411, G05 & \\
\hline Family Lestrigonidae Zeidler, 2004 & & \\
\hline Lestrigonus bengalensis Giles, 1887 & SC82, BH05 & $\begin{array}{l}\text { Bow73, EBW03, } \\
\text { GFG08, PO }\end{array}$ \\
\hline Lestrigonus schizogeneios (Stebbing, 1888) & SC82, Bri86, BH05 & $\begin{array}{l}\text { Hu56 }{ }^{12}, \text { Bow73, } \\
\text { Bru81, OL02, } \\
\text { EBW03, GFG08, } \\
\text { PO }\end{array}$ \\
\hline Lestrigonus crucipes (Bovallius, 1889) & & Bow73, PO \\
\hline Lestrigonus latissimus (Bovallius, 1889) & & Bow73, PO \\
\hline Lestrigonus macrophthalmus (Vosseler, 1901) & SC82, BH05 & $\begin{array}{c}\text { Bow73, LO99, } \\
\text { EBW03, PO }\end{array}$ \\
\hline Lestrigonus schoemakeri Bowman, 1973 & SC82, Bri86, BH05 & $\begin{array}{l}\text { Bow73, Bru81, } \\
\text { OL02, EBW03, } \\
\text { PO }\end{array}$ \\
\hline Phronimopsis spinifera Claus, 1879 & SC82, Bri86, BH05 & OL02, EBW03, PO \\
\hline Themistella fusca (Dana, 1852) & SC82, Bri86, BH05 & Bow73, EBW03, PO \\
\hline Hyperioides longipes Chevreux, 1900 & SC82, Bri86, BH05 & $\begin{array}{l}\text { Hu56, Bow73, } \\
\text { Bru81, OL02, } \\
\text { EBW03 }\end{array}$ \\
\hline Hyperioides sibaginis (Stebbing, 1888) & SC82, Bri86, BH05 & $\begin{array}{l}\text { Bow73, EBW03, } \\
\text { GFG08, PO }\end{array}$ \\
\hline Hyperietta luzoni (Stebbing, 1888) & SC82, BH05 & $\begin{array}{c}\text { Bow73, Bru81, } \\
\text { EBW03, PO }\end{array}$ \\
\hline Hyperietta vosseleri (Stebbing, 1904) & SC82, Bri86, BH05 & $\begin{array}{r}\text { Bow73, Bru81, } \\
\text { EBW03, PO }\end{array}$ \\
\hline Hyperietta stebbingi Bowman, 1973 & SC82, Bri86, BH05 & $\begin{array}{l}\text { Bow73, Bru81, } \\
\text { EBW03 }\end{array}$ \\
\hline Hyperietta stephenseni Bowman, 1973 & SC82, BH05 & Bow73, EBW03, PO \\
\hline Hyperietta parviceps Bowman, 1973 & & $\begin{array}{l}\text { Bow73, Bru81, } \\
\text { OL02, EBW03 }\end{array}$ \\
\hline \multicolumn{3}{|l|}{ Family Iulopidae Zeidler, 2004} \\
\hline Iulopis mirabilis Bovallius, 1887 & & Bru81, EBW03 \\
\hline \multicolumn{3}{|l|}{ Family Dairellidae Bovalllius, 1887} \\
\hline Dairella californica (Bovallius, 1885) & $\mathrm{SC} 82^{13}, \mathrm{BH} 05^{13}$ & $\begin{array}{l}\text { Bru81, OL02, } \\
\quad \text { EBW03 }{ }^{13}, \text { PO }\end{array}$ \\
\hline \multicolumn{3}{|l|}{ Superfamily Lycaepsoidea Bowman \& Gruner, 1973} \\
\hline \multicolumn{3}{|l|}{ Family Lycaepsoidae Chevreux, 1913} \\
\hline Lycaeopsis themistoides Claus, 1879 & SC82, Bri86, BH05 & $\begin{array}{l}\text { OL02, EBW03, } \\
\text { GFG08, PO }\end{array}$ \\
\hline Lycaeopsis zamboangae (Stebbing, 1888) & $\begin{array}{l}\mathrm{SC} 82^{14}, \mathrm{Bri}^{14} 6^{14} \\
\mathrm{BH} 05\end{array}$ & $\begin{array}{c}\text { Hu56, EBW03, } \\
\text { GFG08, PO }\end{array}$ \\
\hline \multicolumn{3}{|l|}{ Superfamily Platysceloidea Bate, 1862} \\
\hline \multicolumn{3}{|l|}{ Family Pronoidae Claus, 1879} \\
\hline Eupronoe maculata Claus, 1879 & SC82, Bri86, BH05 & EBW03 \\
\hline Eupronoe minuta Claus, 1879 & $\mathrm{SC} 82^{15}, \mathrm{BH} 05$ & $\begin{array}{l}\text { Hu56, OL02, } \\
\text { EBW03 }\end{array}$ \\
\hline Eupronoe armata Claus, 1879 & SC82, Bri86, BH05 & EBW03, PO \\
\hline
\end{tabular}


TABLE 1 (continued)

\begin{tabular}{|c|c|c|}
\hline Taxa & $\begin{array}{l}\text { Records from the } \\
\text { Gulf of California }{ }^{a}\end{array}$ & $\begin{array}{l}\text { Records from } \\
\text { the Pacific Coast }{ }^{a}\end{array}$ \\
\hline Pronoe capito Guérin-Méneville, 1836 & SC82, BH05 & EBW03 \\
\hline Parapronoe crustulum Claus, 1879 & $\mathrm{SC} 82^{16}, \mathrm{BH} 05$ & EBW03 \\
\hline Parapronoe parva Claus, 1879 & $\begin{array}{l}\mathrm{Sc} 82^{17}, \text { Sho } 25^{18} \\
\text { BH05 }\end{array}$ & $\begin{array}{l}\mathrm{Hu} 56^{17}, \mathrm{Bru} 81^{17}, \\
\text { OL02, EBW03 } \\
\text { GFG08, PO }\end{array}$ \\
\hline Parapronoe campbelli Stebbing, 1888 & SC82, BH05 & EBW03 \\
\hline Paralycaea gracilis Claus, 1879 & $\mathrm{SC} 82^{20}, \mathrm{BH} 05$ & $\begin{array}{l}\text { Hu56, OL02, } \\
\text { EBW03, GFG08, } \\
\text { PO }\end{array}$ \\
\hline Paralycaea boylei Stebbing, 1888 & SC82, Bri86 & EBW03, PO \\
\hline \multicolumn{3}{|l|}{ Family Anapronoidae Bowman \& Gruner, 1973} \\
\hline \multicolumn{3}{|l|}{ Family Lycaeidae Claus, 1879} \\
\hline Lycaea pulex Marion, 1874 & $\mathrm{BH} 05$ & $\begin{array}{c}\text { OL02, EBW03, } \\
\text { GFG08, PO }\end{array}$ \\
\hline Lycaea pauli Stebbing, 1888 & & EBW03 \\
\hline Lycaea nasuta Claus, 1879 & SC82, BH05 & EBW03 \\
\hline Lycaea serrata Claus, 1879 & SC82, Bri86, BH05 & EBW03, PO \\
\hline Lycaea pachypoda (Claus, 1879) & $\mathrm{SC} 82^{21}, \mathrm{BH} 05$ & OL02, EBW03, PO \\
\hline Lycaea vincentii Stebbing, 1888 & & GFG08, PO \\
\hline Lycaea bajensis Shoemaker, 1925 & Sho25, SC82 & EBW03, PO \\
\hline Lycaea bovalloides Stephensen, 1925 & SC82, Bri86 & EBW03, PO \\
\hline Lycaea bovalli Chevreux, 1900 & & $\mathrm{PO}$ \\
\hline Simorbynchotus antennarius (Claus, 1871) & SC82, Bri86, BH05 & $\begin{array}{l}\text { Bru81, OL02, } \\
\text { EBW03, GFG08, } \\
\text { PO }\end{array}$ \\
\hline \multicolumn{3}{|l|}{ Family Tryphanidae Bovallius, 1887} \\
\hline Tryphana malmi Boeck, 1870 & & $\begin{array}{l}\text { Bru81, OL02, } \\
\text { EBW03 }\end{array}$ \\
\hline \multicolumn{3}{|l|}{ Family Brachyscelidae Stephensen, 1923} \\
\hline Brachyscelus crusculum Bate, 1861 & $\begin{array}{l}\text { Sho25, SC8222, } \\
\text { Bri86, GH04, } \\
\text { BH05 }\end{array}$ & $\begin{array}{c}\text { Bru81, EBW03, } \\
\text { GFG08, PO }\end{array}$ \\
\hline Brachyscelus globiceps (Claus, 1879) & SC82, Bri86, BH05 & EBW03, PO \\
\hline Brachyscelus rapax (Claus, 1879) & BH05 & EBW03 \\
\hline Brachyscelus rapacoides Stephensen, 1925 & SC82 & EBW03, PO \\
\hline Euthamneus rostratus (Bovallius, 1887) & $\begin{array}{l}\mathrm{SC}^{23}, \mathrm{~B}^{23} 86^{23} \\
\mathrm{GH} 04, \mathrm{BH} 05\end{array}$ & EBW03, PO \\
\hline \multicolumn{3}{|l|}{ Family Oxycephalidae Bate, 1861} \\
\hline Oxycephalus piscator Milne-Edwards, 1830 & SC82, Bri86, BH05 & EBW03 \\
\hline Oxycephalus clausi Bovallius, 1887 & $\begin{array}{l}\text { SC82, Bri86, GH04, } \\
\text { BH05 }\end{array}$ & $\begin{array}{c}\text { OL02, EBW03, } \\
\text { GFG08, PO }\end{array}$ \\
\hline Oxycephalus latirostris Claus, 1889 & & EBW03 \\
\hline Streetsia challengeri Stebbing, 1888 & SC82, BH05 & $\begin{array}{l}\text { Bru81, OL02, } \\
\text { EBW03 }\end{array}$ \\
\hline Streetsia steenstrupi (Bovallius, 1887) & $\mathrm{SC} 82, \mathrm{BH} 05$ & Bru81, EBW03 \\
\hline Streetsia porcella (Claus, 1879) & & Bru81, EBW03, PO \\
\hline Streetsia mindanaonis Stebbing, 1888 & $\mathrm{SC} 82, \mathrm{BH} 05$ & Bru81, EBW03, PO \\
\hline Leptocotis tenuirostris (Claus, 1871) & SC82, BH05 & EBW03, PO \\
\hline Calamorbynchus pellucidus Streets, 1878 & $\mathrm{SC} 82, \mathrm{BH} 05$ & EBW03 \\
\hline Glossocephalus milneedwardsi Bovallius, 1887 & SC82, BH05 & $\begin{array}{l}\text { Bru81, EBW03, } \\
\text { GFG08, PO }\end{array}$ \\
\hline Cranocephalus scleroticus (Streets, 1878) & SC82, BH05 & Bru81, EBW03, PO \\
\hline Rhabdosoma armatum (Milne-Edwards, 1840) & $\mathrm{SC} 82, \mathrm{BH} 05$ & EBW03 \\
\hline Rhabdosoma whitei Bate, 1862 & SC82, Bri86, BH05 & $\begin{array}{l}\text { S1878, EBW03, } \\
\text { GFG08, PO }\end{array}$ \\
\hline Rhabdosoma brevicaudatum Stebbing, 1888 & $\mathrm{SC} 82, \mathrm{BH} 05$ & EBW03 \\
\hline Rhabdosoma minor Fage, 1954 & $\mathrm{SC} 82, \mathrm{BH} 05$ & EBW03, PO \\
\hline
\end{tabular}


TABLE 1 (continued)

\begin{tabular}{|c|c|c|}
\hline Taxa & $\begin{array}{l}\text { Records from the } \\
\text { Gulf of California }{ }^{a}\end{array}$ & $\begin{array}{l}\text { Records from } \\
\text { the Pacific Coast }{ }^{a}\end{array}$ \\
\hline \multicolumn{3}{|l|}{ Family Platyscelidae Bate, 1862} \\
\hline Platyscelus ovoides (Risso, 1816) & & EBW03 \\
\hline Platyscelus serratulus Stebbing, 1888 & $\begin{array}{c}\mathrm{Sho}^{25^{24}}, \mathrm{SC} 82, \\
\text { Bri86, BH05 }\end{array}$ & Hu56, EBW03, PO \\
\hline Platyscelus crustulatus (Claus, 1879) & & $\mathrm{PO}$ \\
\hline Hemityphis tenuimanus Claus, 1879 & $\begin{array}{l}\mathrm{SC}^{\mathrm{S}} 2^{25}, \mathrm{Bri}^{26} 6^{26} \\
\mathrm{BH} 05^{25}\end{array}$ & EBW0325,27 \\
\hline Paratyphis maculatus Claus, 1879 & SC82, BH05 & EBW03 \\
\hline Paratyphis parvus Claus, 1887 & & $\mathrm{PO}$ \\
\hline Paratyphis spinosus Spandl, 1924 & SC82, BH05 & EBW03 \\
\hline Tetratbyrus forcipatus Claus, 1879 & $\begin{array}{l}\text { Sho2 } 5^{28}, \text { SC82, } \\
\text { BH } 05\end{array}$ & $\begin{array}{c}\text { Bru81, EBW03, } \\
\text { GFG08, PO }\end{array}$ \\
\hline Tetrathyrus arafurae Stebbing, 1888 & SC82, BH05 & EBW03 \\
\hline Amphithyrus bispinosus Claus, 1879 & SC82, Bri86, BH05 & $\begin{array}{l}\text { EBW03, GFG08, } \\
\text { PO }\end{array}$ \\
\hline Amphithyrus similis Claus, 1879 & SC82, BH05 & EBW03 \\
\hline Amphithyrus muratus Volkov, 1982 & & $\mathrm{PO}$ \\
\hline Amphitbyrus sculpturatus Claus, 1879 & $\begin{array}{c}\text { Sho } 25^{29}, \text { SC } 82^{29} \\
\text { Bri86, BH05 }\end{array}$ & EBW03, PO \\
\hline \multicolumn{3}{|l|}{ Family Parascelidae Claus, 1879} \\
\hline Schizoscelus ornatus Claus, 1879 & $\mathrm{SC} 82, \mathrm{BH} 05$ & EBW03 \\
\hline Thyropus sphaeroma (Claus, 1879) & SC82, BH05 & EBW03, PO \\
\hline Thyropus similis (Stephensen, 1925) & SC82 & \\
\hline Parascelus edwardsi Claus, 1879 & $\begin{array}{l}\text { Sho } 25^{30}, \mathrm{SC} 82^{31}, \\
\text { Bri86 } \\
\text { BH } 05^{32}, \mathrm{GH} 04^{33}\end{array}$ & $\begin{array}{l}\mathrm{Hu} 56^{33}, \mathrm{Bru} 81^{31} \\
\text { OL02, EBW03 } \\
\text { GFG08, PO }\end{array}$ \\
\hline
\end{tabular}

a Abbreviations of the works included in this analysis: Bow60, Bowman (1960); Bow73, Bowman (1973); Bow78, Bowman (1978); Bri86, Brinton et al. (1986); Bru81, Brusca (1981); BH05, Brusca and Hendrickx (2005); EBW03, Escobar-Briones and Winfield (2003); GH04, Gasca and Haddock (2004); G05, Gasca (2005); GFG08, Gasca and Franco-Gordo (2008); Hu56, Hurley (1956); OL02, Ohman and Lavaniegos (2002); PO, pers. obs.; SC82, Siegel-Causey (1982); SH03, Shih and Hendrycks (2003); Sho25, Shoemaker (1925); S1878, Streets (1878).

Notes: 1, Probably L. loveni loveni; 2, as Lanceola aestiva Stebbing, 1888 (see Vinogradov et al. [1996]); 3, as Vibilia californica Holmes, 1908 (see Zeidler [2003a]); 4, as Vibilia wolterecki Behning, 1939 (see Zeidler [2003a]); 5, also as Vibilia wolterecki Behning, 1939 (see Zeidler [2003a]); 6, as Phronima bucephala Giles, 1877 (see Shih [1991]); 7, as Phronima stebbingi Vosseler, 1901 (see Shih [1991]); 8, as the bystrix form by Bovallius, 1889; 9, arctic-boreal form, regional records are doubtful (see Vinogradov et al. [1996]); 10, as Parathemisto pacifica Stebbing, 1888 (see Zeidler [2004b]); 11, as Hyperoche medusarum (Kröyer, 1838) (see Gasca [2005]); 12, as Hyperia bengalensis (Giles) (see Brusca [1981]); 13, as D. californica Bovallius, 1887 and D. latissima Bovallius, 1887 (see Zeidler [2004b]); 14, also as Lycaeopsis neglecta Pirlot, 1929 and L. pauli Stebbing, 1888 (see Zeidler [2004a]; 15, also as Eupronoe pacifica Stebbing, 1888 (see Vinogradov et al. [1996]); 16, also as Parapronoe clausioides Stebbing, 1888 (see Vinogradov et al. [1996]); 17, as Sympronoe parva Stebbing, 1888 (see Vinogradov et al. [1996]); 18, as Sympronoe anomala Shoemaker, 1925 (see Vinogradov et al. [1996]); 19, as Parapronoe parva parva Claus, 1879 and Parapronoe parva septenarticulata (Stephensen, 1925) (see Zeidler [1998]); 20, also as Paralycaea newtoniana Bovallius, 1887 (see Harbison et al. [1977]); 21, as Pseudolycaea pachypoda Claus, 1879 (see Vinogradov et al. [1996]); 22, also as $B$. acuticaudatus Stebbing, 1888 (see Vinogradov et al. [1996]); 23, as Thamneus platyrrynchus Stebbing, 1888 (see Vinogradov et al. [1996]); 24, as P. dubius Shoemaker, 1925 (see Vinogradov et al. [1996]); 25, as Tetrathyrus pulchellus Barnard, 1930 and as Hemityphys rapax (Milne-Edwards, 1930) (see Zeidler [1996]); 26, as Tetrathyrus pulchellus Barnard, 1930 (see Zeidler [1996]); 27, as Hemiscelus diplochelatus Stewart, 1913 (see Zeidler [1998]); 28, as T. sanctijosephi Shoemaker, 1925 (see Vinogradov et al. [1996]); 29, as A. orientalis Stebbing (see Vinogradov et al. [1996]); 30, as P. zebu Stebbing (see Zeidler [1998]); 31, as Thyrophus edwardsi (Claus, 1879) and T. thyphoides (Claus, 1879) (see Zeidler [1998]); 32, as T. edwardsi (Claus, 1879) (see Zeidler [1998]); 33, as P. thyphoides Claus, 1879 (see Zeidler [1998]); 34, also as P. thyphoides Claus, 1879 (see Zeidler [1998]).

Up to seven species have not been included in previous accounts of the Mexican Pacific, including the most recent one by Brusca and Hendrickx (2005) (Table 1). Two of these species, Lycaea vincentii (ECO-CHZ 03042) and Hyperoche picta (ECO-CHz 03542), have been recorded previously but from adjacent waters of the Pacific and were recorded off the coasts of the Mexican state of Jalisco (Gasca and Franco-Gordo 2008; pers. obs.). The record of Vibilia borealis (ECO-CHZ 03126) is regarded as new in the MP although 
the species was mentioned by EscobarBriones and Winfield (2003), but there is no evidence of the source supporting this record. The remaining four species, Lycaea bovalli (eCO-CHz 03148), Platyscelus crustulatus (eCOCHZ 03545), Paratyphis parvus (ECO-CHZ 03099), and Amphythyrus muratus (ECO-CHZ 03110), are new records in the Mexican waters of the Pacific (pers. obs).

Based on the available data, the Gulf of California harbors the highest number of species (114) recorded in Mexican waters of the Pacific, whereas the northern sector of the Pacific coast (north from $22^{\circ} 52^{\prime} \mathrm{N}$ ) off Baja California has fewer species (87); the central sector of the Mexican tropical Pacific zone is known to harbor 68 species. There are only six records of hyperiids from the southernmost areas of the Mexican tropical Pacific, off the coasts of Guerrero, Oaxaca, and Chiapas (Figure 1).

A comparative analysis of the records within the area showed that up to 75 species are found in both the Gulf of California and off the Baja California coast. There are 30 species that have been recorded from the gulf only, and another group includes 30 species that have been found only on the Pacific coast north from $17^{\circ} 50^{\prime} \mathrm{N}$. The gulf and the tropical sectors of the Mexican Pacific coast share eight species only; five species have been reported exclusively from the tropical Pacific area.

\section{Taxonomic Remarks}

The current taxonomy of the Hyperiidea is highly dynamic, and several changes have taken place as a result of recent revision works in different families (i.e., Zeidler 2003a,b, 2004a,b, 2006). Many of the oldest regional names/records have been changed; for instance, only three (Anchylomera blossevillei, Lycaea bajensis, and Brachyscelus crusculum) of the nine nominal species mentioned by Shoemaker (1925) retained the name originally used by the author (Table 1). The analysis of the records in the MP and the synonymies of the species indicate that up to 31 species recorded in the literature examined (Table 1) are invalid names and thus were excluded from the updated account but were included with their corrected names. Some of the invalid names were retained in several contributions and even in the most recent accounts (i.e., Brusca 1981, Siegel-Causey 1982, Escobar-Briones and Winfield 2003, Brusca and Hendrickx 2005). Overall, the number of invalid names represents $21 \%$ of the previous, unrevised lists.

There are some interesting records that are commented upon in more detail here. One of these is Lycaea bajensis, originally described from the Gulf of California by Shoemaker (1925); it was soon synonymized to L. pulex by Hurley (1956) and Vinogradov et al. (1996) and successively included as such in the regional listings (Hurley 1956, Brusca and Hendrickx 2005). However, from the taxonomic revision of the genus by Harbison and Madin (1976), it was considered as a separate, valid species. It has been recorded in the area only twice after its description (Siegel-Causey 1982, Gasca and FrancoGordo 2008) but was reported from other geographical areas including Australian waters (Zeidler 1998) and the Sargasso Sea (Gasca 2007). Local records of L. pulex in the region could refer to either of three morphologically similar species, L. pulex, L. bajenis, and L. vincentii; hence, individual records should be checked (Gasca and Franco-Gordo 2008). Other species with taxonomic complexities are Parascelus edwardsi and Hemityphis tenuimanus, in reference to which several other different names have been used in the literature (see Table 1).

In the checklist by Escobar-Briones and Winfield (2003), some genera and species (Vibilia armata, V. australis, Pbronima curvipes, P. dunbari) are misplaced in families to which they do not belong (i.e., Synopyiidae, Lanceolidae, and Phronimidae). They also included a record of Oxycephalus pellucidus Streets, 1878; however, this binomen has not been assigned to any known species. Another oxycephalid, Calamorbynchotus pellucidus Streets, 1878 , is currently valid and was included as such by Escobar-Briones and Winfield (2003).

All the entries of species whose taxonomic status or name have changed are referred to 
footnotes in Table 1. Some of the records included are from literature sources only, and the original ones are not explicitly stated (see Table 1); hence, these particular records are not confirmed for the MP (i.e., Mimonectes diomedae, Vibilia borealis, and Lanceola loveni grossipes) and thus should be reexamined.

\section{DISCUSSION}

The most diverse infraorder in this account, the Physocephalata, is represented in the MP by a diverse array of 119 species; these are mostly epipelagic forms, whereas the order Physosomata, predominantly deep-living, is represented by 31 species only. This difference is attributed to the emphasis given to sampling the upper layers $(0-200 \mathrm{~m})$ in the region, which have been surveyed intensely in most instances; relatively less attention has been given to the meso- and bathypelagic zooplankton fauna. Only a few surveys have reached these layers (i.e., Hurley 1956, Gasca and Haddock 2004). Currently, the deepliving Physosomata represent approximately $38 \%$ of the known species of the order Hyperiidea. In this checklist they accounted for $21 \%$ of the species recorded in the MP, and more than half of these are from the Gulf of California, where the deep-living hyperiid fauna is better known. The diversity of this group in the region could have been underestimated as a result of the sampling methods used. Future research in the area should emphasize the subsurface layers; these efforts should yield many new records of the hyperiid fauna, particularly in the tropical Pacific.

The latitudinal distribution of the number of species recorded in the MP is asymmetrical when comparing the different sectors. The gulf area, together with the Pacific coast off Baja California, harbors the highest number of species records (136 species). This is not related to a latitudinal diversity gradient but is clearly a result of the greater sampling effort in the California Current and the Gulf of California. Despite this general trend in the California subregions, surveys on the hyperiids are relatively scarce but enough to make a sharp difference with respect to the adjacent tropical areas of the MP. The cen- tral part of the MP, south of the Gulf of California from $22^{\circ} 52^{\prime} \mathrm{N}$, had a noticeable decrease in the number of species of hyperiids recorded (68 species); the hyperiid fauna of this area remained almost completely unknown for many decades after the four species recorded by Hurley (1956) ( $V$. armata, $V$. viatrix, A. blossevillei, and Parapronoe parva) off the coasts of Michoacán $\left(17^{\circ} 50^{\prime} \mathrm{N}\right)$ and Vibilia cultripes from off the coast of Colima $\left(\begin{array}{lll}16^{\circ} & 53^{\prime} \mathrm{N}\end{array}\right)$ (Shih and Hendrycks 2003). Most of the records presented herein from this central sector resulted from recent surveys in shelf and oceanic areas off the states of Jalisco and Nayarit (Gasca and FrancoGordo 2008) and personal observations from samples obtained off Jalisco and Colima. Clearly, the least-known hyperiid fauna in the Mexican waters of the Pacific is that of the lowest latitudes, south from $17^{\circ} 50^{\prime} \mathrm{N}$, off the coasts of three Mexican states, to the border with Guatemala $\left(14^{\circ} 31^{\prime} \mathrm{N}\right)$; there are only six records from this area, all of the genus Vibilia (Shih and Hendrycks 2003).

The species recorded as the most abundant in the Gulf of California are Lestrigonus bengalensis, Primno brevidens, L. shoemakeri, Vibilia armata, and Thyropus edwardsi (Siegel-Causey 1982). In the California Current zone off Baja California, the group of most abundant species is somewhat different, with $P$. brevidens, Paraphronima gracilis, V. armata, Pbronimopsis spinifera, Phronima sedentaria, $L$. schizogeneios, and L. shoemakeri being among the most abundant (Lavaniegos and Ohman 1999). In the tropical part of the Mexican $\mathrm{Pa}$ cific the group of dominant species is also different: L. bengalensis and Hyperioides sibaginis (Gasca and Franco-Gordo 2008). Similar variations at a subregional scale were detected by Vinogradov (1991) from the Southern $\mathrm{Pa}$ cific Gyre. According to Vinogradov (1999), any part of the Pacific Ocean harbors a distinct group of species that includes Phronima atlantica, Phronimella elongata, Phrosina semilunata, a species of Primno, Scina crassicornis, Anchylomera blossevillei, Hemityphis tenuimanus, Vibilia armata, and a species of Brachyscelus (Vinogradov 1999). A closer analysis of the regional hyperiid composition shows that each subregion has a distinct array of species 
and confirms the need of studying the local faunas in more detail to complete the regional profile of diversity patterns in the group.

The number of species recorded from the Mexican waters of the eastern Pacific indicates that this area is now among the best studied in the region; approximately $68 \%$ of the known species from the Pacific Ocean (Vinogradov et al. 1996) occur in the MP. The 68 species recorded from the tropical areas of the MP represent nearly $40 \%$ of the species known from the tropical Pacific (Vinogradov 1991) and show a high affinity with those of other tropical areas of the $\mathrm{Pa}$ cific; nearly $95 \%$ of the species recorded from the South Pacific Gyre, also a fully tropical system (Vinogradov 1991), have been recorded in the MP.

Overall, species richness from the MP is comparable with that known from other subregions of the Pacific Ocean, including the Southern Gyre (Vinogradov 1991: 119 species) and the North Pacific Gyre (Shulenberger 1977: 83 species). The number of species from the tropical areas is still low when compared with accounts from other tropical areas of the Mexican seas; for instance, ca. 100 species have been recorded in the Gulf of Mexico (LeCroy et al. 2008) and 62 from the western Caribbean Sea (Gasca and Shih 2001, 2003, Gasca and SuárezMorales 2004). The hyperiid fauna of these large, unstudied areas of the southern MP are likely to yield important information to reveal the latitudinal patterns of diversity along the eastern Pacific.

\section{ACKNOWLEDGMENTS}

Relevant literature was kindly provided by Rebecca Scheinberg, Monterey Bay Aquarium Research Institute, and Lana Ong, National Museum of Natural History, Smithsonian Institution. The comments from two anonymous reviewers greatly improved an earlier version of this work.

\section{Literature Cited}

Bowman, T. E. 1960. The pelagic amphipod genus Parathemisto (Hyperiidea: Hyperii- dae) in the North Pacific and adjacent Arctic Ocean. Proc. U.S. Natl. Mus. 112:343-392.

. 1973. Pelagic amphipods of the genus Hyperia and closely related genera (Hyperiidea: Hyperiidae). Smithson. Contrib. Zool. 136:1-76.

. 1978. Revision of the pelagic amphipod genus Primno (Hyperiidea: Phrosinidae). Smithson. Contrib. Zool. 275: $1-23$.

Brinton, E., A. Fleminger, and D. SiegelCausey. 1986. The temperate and tropical planktonic biotas of the Gulf of California. Calif. Coop. Oceanic Fish. Invest. Rep. 27:228-266.

Brusca, G. J. 1967. The ecology of pelagic amphipods. I. Species accounts, vertical zonation and migration of amphipods from the waters off southern California. Pac. Sci. 21:382-393.

. 1981. Annotated keys to the Hyperiidea (Crustacea: Amphipoda) of North American coastal waters. Allan Hancock Found. Tech. Rep. 5:1-76.

Brusca, R. C., and M. E. Hendrickx. 2005. Crustacea 4. Peracarida: Lophogastrida, Mysida, Amphipoda, Tanaidacea and Cumacea. Pages 147-154 in M. E. Hendrickx, R. C. Brusca, and L. T. Findley, eds. A distributional checklist of the macrofauna of the Gulf of California, Mexico. Part I. Invertebrates. Arizona-Sonora Desert Museum, Tucson, Arizona.

Escobar-Briones, E., and I. Winfield. 2003. Patrones de la riqueza de especies y evolución del registro taxonómico de anfípodos del Pacífico en el territorio mexicano. Pages 1-19 in M. E. Hendrickx, ed. Contribuciones al estudio de los crustáceos del Pacífico Este. Vol. 2. Instituto de Ciencias del Mar y Limnología, Universidad Nacional Autónoma de México, Mexico City.

Escobar-Briones, E., I. Winfield, M. Ortíz, R. Gasca, and E. Suárez-Morales. 2002. Orden Amphipoda (Crustacea: Peracarida). Pages 341-371 in J. Llorente-Bousquets and J. Morrone, eds. Biodiversidad, taxonomía y biogeografía de artrópodos de México. Vol. 3. Comisión Nacional para el Conocimiento y Uso de la Biodiversidad/ 
Universidad Nacional Autónoma de México/Bayer, Mexico.

Gasca, R. 2005. Hyperoche shibi sp. nov. (Crustacea: Peracarida: Amphipoda), a symbiont of a deep-living medusa in the Gulf of California. J. Plankton Res. 27:617-621.

. 2007. Hyperiid amphipods of the Sargasso Sea. Bull. Mar. Sci. 81:115-125.

Gasca, R., and C. Franco-Gordo. 2008. Hyperiid amphipods (Peracarida) from Banderas Bay, Mexican tropical Pacific. Crustaceana (Leiden) 81:563-575.

Gasca, R., and S. H. Haddock. 2004. Associations between gelatinous zooplankton and hyperiid amphipods (Crustacea: Peracarida) in the Gulf of California. Hydrobiologia 530/531:529-535.

Gasca, R., and C.-T. Shih. 2001. Hyperiid amphipods of surface waters of the western Caribbean Sea (1991). Crustaceana (Leiden) 74:489-499.

. 2003. Hyperiid amphipods of Banco Chinchorro. Bull. Mar. Sci. 73:91-98.

Gasca, R., and E. Suárez-Morales. 2004. Distribution and abundance of hyperiid amphipods (Crustacea: Peracarida) from the Mexican Caribbean Sea (August 1986). Caribb. J. Sci. 40:23-30.

Harbison, G. R., D. C. Bigs, and L. P. Madin. 1977. The associations of Amphipoda Hyperiidea with gelatinous zooplankton-II. Associations with Cnidaria, Ctenophora and Radiolaria. Deep-Sea Res. 24:465-468.

Harbison, G. R., and L. P. Madin. 1976. Description of the female Lycaea nasuta Claus, 1879 with an illustrated key to the species of Lycaea Dana, 1852 (Amphipoda, Hyperiidea). Bull. Mar. Sci. 26:165-171.

Hurley, D. E. 1956. Bathypelagic and other Hyperiidea from California waters. Allan Hancock Found. Occas. Pap. 18:1-25.

Laval, P. 1980. Hyperiid amphipods as crustacean parasitoids associated with gelatinous zooplankton. Oceanogr. Mar. Biol. Annu. Rev. 18:11-56.

Lavaniegos, B. E., and M. D. Ohman. 1999. Hyperiid amphipods as indicators of climate change in the California Current. Pages 489-509 in F. R. Schram and J. C. von Vaupel Klein, eds. Crustaceans and the biodiversity crisis. Proceedings, 4th International Crustacean Congress, Amsterdam. Brill, Leiden.

LeCroy, S., R. Gasca, I. Winfield, M. Ortiz, and E. Escobar-Briones. 2008. Amphipoda (Crustacea) of the Gulf of Mexico. Pages 1824-1898 in D. L. Felder and D. K. Camp, eds. Gulf of Mexico-Origins, waters, and biota. Biodiversity. Texas A\&M University Press, College Station.

Ohman, M. D., and B. E. Lavaniegos. 2002. Comparative zooplankton sampling efficiency of a ring net and Bongo net with comments on pooling of subsamples. Calif. Coop. Oceanic Fish. Invest. Rep. 42:162-173.

Shih, C.-T. 1991. Description of two new species of Phronima Latreille, 1802 (Amphipoda: Hyperiidea) with a key to all species of the genus. J. Crustacean Biol. 11:322-335.

Shih, C.-T., and Q.-C. Cheng. 1995. Zooplankton of China seas (2). The Hyperiidea (Crustacea: Amphipoda). China Ocean Press, Bejing.

Shih, C.-T., and E. A. Hendrycks. 2003. A new species and new records of the genus Vibilia Milne Edwards, 1830 (Amphipoda: Hyperiidea: Vibiliidae) occurring in the eastern Pacific Ocean. J. Nat. Hist. 37:253-296.

Shoemaker, C. R. 1925. The Amphipoda collected by the United States Fisheries Steamer "Albatross" in 1911, chiefly in the Gulf of California. Bull. Am. Mus. Nat. Hist. 52:21-61.

Shulenberger, E. 1977. Hyperiid amphipods from the zooplankton community of the North Pacific central gyre. Mar. Biol. (Biol.) 42:375-385.

Siegel-Causey, D. 1982. Factors determining the distribution of hyperiid Amphipoda in the Gulf of California. Ph.D. thesis, University of Arizona, Tucson.

Streets, T. H. 1878. Pelagic Amphipoda. Proc. Acad. Nat. Sci. Phila. (1878): 276291, pl. 2.

Vinogradov, G. M. 1991. Hyperiid amphipods in the eastern part of the South $\mathrm{Pa}$ cific gyre. Mar. Biol. (Berl.) 109:259-265. . 1999. Amphipoda. Pages 1141-1240 
in D. Boltovskoy, ed. South Atlantic zooplankton. Backhuys Publishers, Leiden.

Vinogradov, M. E., A. F. Volkov, and T. N. Semenova. 1996. Hyperiid amphipods (Amphipoda, Hyperiidea) of the world oceans. Science Publishers, Inc. Lebanon, New Hampshire.

Zeidler, W. 1996. On the identity of Typhis $\operatorname{rapax}$ H. Milne Edwards, 1830 (Amphipoda, Hyperiidea). Crustaceana (Leiden) 69:734-741.

1998. Pelagic amphipods (Crustacea: Amphipoda: Hyperiidea) collected from eastern and south-eastern Australian waters by the CSIRO research vessel "Warreen" during the years 1938-1941. Rec. S. Aust. Mus. (Adelaide) Monogr. Ser. 4:1-143.

1999. Review of the hyperiidean amphipod genus Oxycephalus Milne-Edwards (Crustacea: Amphipoda: Hyperiidea: Oxycephalidae). Invertebr. Taxon. 13:391424. 2003a. A review of the hyperiidean amphipod superfamily Vibilioidea Bowman and Gruner, 1973 (Crustacea: Amphipoda: Hyperiidea). Zootaxa 280:1-104.

- 2003b. A review of the hyperiidean amphipod family Cystisomatidae Willemoes-Suhm, 1875 (Crustacea: Amphipoda: Hyperidea). Zootaxa 141:1-43.

P 2004a. A review of the hyperiidean amphipod superfamily Lycaepsoidea Bowman and Gruner, 1973 (Crustacea: Amphipoda: Hyperiidea). Zootaxa 520:1184.

. 2004b. A review of the families and genera of the hyperiidean amphipod superfamily Phronimoidea Bowman and Gruner, 1973 (Crustacea: Amphipoda: Hyperiidea). Zootaxa 567:1-66.

2006. A review of the hyperiidean amphipod superfamily Archaeoscinoidea Vinogradov, Volkov and Semenova, 1982 (Crustacea: Amphipoda: Hyperiidea). Zootaxa 1125:1-37. 
